\title{
Nuclear shell effects near the r-process path *
}

\author{
M.M. Sharma and A.R. Farhan ${ }^{\text {a }}$ \\ aPhysics Department, Kuwait University, P.O. Box 5969, Safat, Kuwait 13060
}

We have studied the evolution of the shell structure of nuclei near the neutron drip line in the Relativistic Hartree-Bogoliubov (RHB) theory with the vector self-coupling of $\omega$ meson. The experimental data on the shell effects about the waiting-point nucleus ${ }^{80} \mathrm{Zn}$ are reproduced successfully. It is shown that the shell effects at $N=82$ near the r-process path remain strong. A quenching exhibited by the $\mathrm{HFB}+\mathrm{SkP}$ approach is shown not to be compatible with the available data.

\section{INTRODUCTION}

The $N=82$ nuclei at r-process path are assumed to play a significant role in providing nuclear abundances about $A \sim 130$ [1]. Since nuclei contributing to this peak are extremely neutron-rich and are not accessible experimentally, there prevail conflicting view points [2,3] on the strength of the shell effects near the neutron drip line. In this work, we examine how the shell effects evolve with isospin in the region of the astrophysically important magic number $N=82$ near the neutron drip line. Using the experimental data on the waiting-point nucleus ${ }^{80} \mathrm{Zn}(N=50)$, we explore the shell effects near the $\mathrm{r}$ process path in the framework of the Relativistic Hartree-Bogoliubov (RHB) theory with the vector self-coupling of $\omega$ meson. In this approach, the shell effects about the stability line have been described successfully [4].

\section{THE RMF AND THE RHB APPROACH}

The RMF Lagrangian describes the nucleons as Dirac spinors moving in meson fields and is given by

$$
\begin{aligned}
\mathcal{L}= & \bar{\psi}\left(\not p-g_{\omega} \psi-g_{\rho} \vec{p} \vec{\tau}-\frac{1}{2} e\left(1-\tau_{3}\right) A-g_{\sigma} \sigma-M_{N}\right) \psi \\
& +\frac{1}{2} \partial_{\mu} \sigma \partial^{\mu} \sigma-U(\sigma)-\frac{1}{4} \Omega_{\mu \nu} \Omega^{\mu \nu}+\frac{1}{2} m_{\omega}^{2} \omega_{\mu} \omega^{\mu} \\
& +\frac{1}{2} g_{4}\left(\omega_{\mu} \omega^{\mu}\right)^{2}-\frac{1}{4} \vec{R}_{\mu \nu} \vec{R}^{\mu \nu}+\frac{1}{2} m_{\rho}^{2} \vec{\rho}_{\mu} \vec{\rho}^{\mu}-\frac{1}{4} F_{\mu \nu} F^{\mu \nu}
\end{aligned}
$$

where $M_{N}$ is the bare nucleon mass and $\psi$ is its Dirac spinor. In addition, we have the scalar meson $(\sigma)$, isoscalar vector mesons $(\omega)$, isovector vector mesons $(\rho)$ and the photons $A^{\mu}$, with the masses $m_{\sigma}, m_{\omega}$ and $m_{\rho}$ and the coupling constants $g_{\sigma}, g_{\omega}, g_{\rho}$, respectively.

\footnotetext{
*This work is supported by the Project No. SP056 of the Research Administration, Kuwait University.
} 
A nonlinear scalar potential $U(\sigma)=\frac{1}{2} m_{\sigma}^{2} \sigma^{2}+\frac{1}{3} g_{2} \sigma^{3}+\frac{1}{4} g_{3} \sigma^{4}$ for $\sigma$-mesons is used for a realistic description of finite nuclei. Recently, we have also added the non-linear vector self-coupling of $\omega$-meson [6] which is represented by $g_{4}$.

For nuclei near a drip line, a coupling to the continuum is required. A self-consistent treatment of pairing is also necessary. We employ the framework of RHB theory which takes into account both these features. The corresponding relativistic Dirac-HartreeBogoliubov (RHB) equations are obtained as

$$
\left(\begin{array}{cc}
h & \Delta \\
-\Delta^{*} & -h^{*}
\end{array}\right)\left(\begin{array}{c}
U \\
V
\end{array}\right)_{k}=E_{k}\left(\begin{array}{c}
U \\
V
\end{array}\right)_{k},
$$

where $E_{k}$ are quasiparticle energies and the coefficients $U_{k}$ and $V_{k}$ are four-dimensional Dirac spinors. The pairing potential $\Delta$ in Eq. (2) is given by

$\Delta_{a b}=\frac{1}{2} \sum_{c d} V_{a b c d}^{p p} \kappa_{c d}$

The RHB calculations are performed by expanding fermionic and bosonic wavefunctions in 20 oscillator shells for a spherical configuration. We have used the force NL-SH [7] with the non-linear scalar self-coupling and the forces NL-SV1 and NL-SV2 with both the scalar and the vector self-couplings. The forces NL-SV1 and NL-SV2 have recently been developed in order to soften the high-density equation of state of nuclear matter and are shown to improve the ground-state properties of nuclei [4, 8].

\section{RESULTS AND DISCUSSION}

In our previous work, it was shown that the existing nuclear forces based upon the nonlinear scalar self-coupling of $\sigma$-meson exhibit shell effects which were stronger than suggested by the experimental data. This problem was solved by introducing the nonlinear vector self-coupling of $\omega$-meson in the RHB theory [4]. Having our working basis established, we explore the shell effects about ${ }^{80} \mathrm{Zn}(N=50) .{ }^{80} \mathrm{Zn}$ is a waiting point nucleus and here the r-process path comes closest to the $\beta$-stability line. We show in Fig. 1 the results on $S_{2 n}$ values for the Zn isotopes. The kink at $N=50$ (Fig 1.a) shows that NL-SH (scalar self-coupling only) exhibits shell effects which are much stronger than the experimental data. In comparison, the shell gap with NL-SV2 (both the scalar and vector self-couplings) [四] is reduced as compared to NL-SH. The shell gap is, however, still larger than the experimental one. In Fig. 1(b) we show the $S_{2 n}$ values obtained with the force NL-SV1. The slope of the kink shows that NL-SV1 is able to reproduce well the empirical shell effects in the waiting point region. A comparison with the HFB+SkP results shows that the shell effects with SkP are strongly quenched. The shell effects were shown to be quench with $\mathrm{SkP}$ already at the stability line [4. This behaviour is evidently due to the high effective mass $m^{*} \sim 1$.

With the formalism established in [4] and tested in Fig. 1, we extend it to explore the inaccessible region of the neutron drip-line about $N=82$. In order to visualize the evolution of shell effects, we have chosen the isotopic chains of $\mathrm{Kr}$, Sr, Zr and Mo $(Z=36-42)$. The results with RHB using various forces are shown in Fig. 2. For a given force, the shell gap at $N=82$ shows a steady decrease in moving from Mo (a) to 


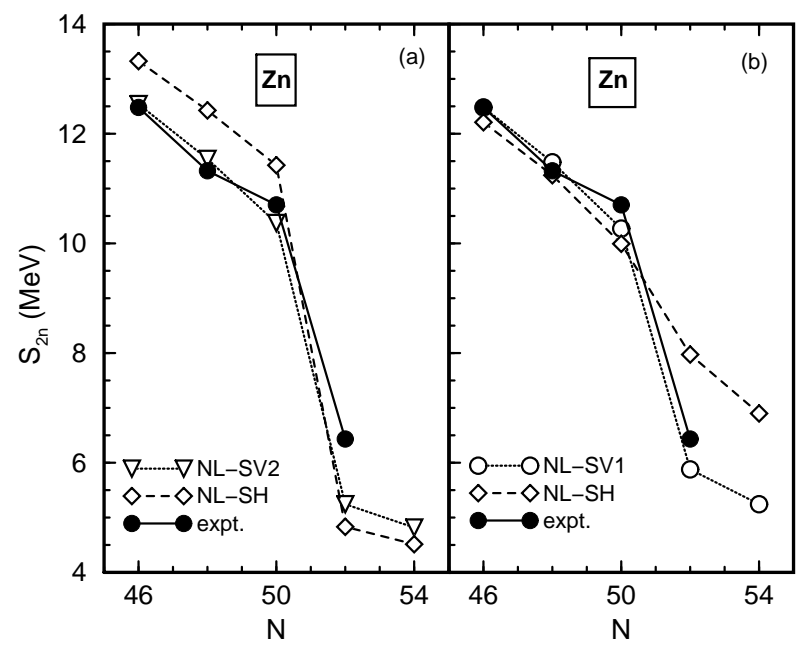

Figure 1. $S_{2 n}$ values for $\mathrm{Zn}$ isotopes in the RHB theory.

Kr (d). Nuclei become increasingly unbound and a coupling to the continuum arises in going to larger neutron to proton ratios. The results with the non-linear scalar coupling (NL-SH) show a shell gap at $N=82$, which is largest amongst all the forces. Similarly strong shell effects with NL-SH were also shown for $N=82$ nuclei near the drip line in ref. [2] within the BCS pairing. With the force NL-SV2 with the vector self-coupling of $\omega$-meson, the shell effects are milder as compared to NL-SH for all the chains. The results (Fig. 2) with our benchmark force NL-SV1 show that the shell gap at $N=82$ is reduced as compared to NL-SV2. This is again similar to that observed for the Zn isotopes. However, the shell effects with NL-SV1 are still stronger as compared to the $\mathrm{HFB}+\mathrm{SkP}$ results as shown in the figure. This is especially true for Mo and Zr isotopes at $\mathrm{N}=82$, where the $\mathrm{r}$-process path is assumed to pass through. The quenching shown by $\mathrm{SkP}$ in this region is as expected. This is consistent with a strong quenching exhibited by $\mathrm{HFB}+\mathrm{SkP}$ at the stability line [4] and also near the waiting-point nucleus ${ }^{80} \mathrm{Zn}$ as shown in Fig. 1(b). In comparison, the RHB approach with NL-SV1 shows stronger shell effects about the stability line [4] as well as in the waiting-point region at $N=50$, which is consistent with the experimental data.

The shell effects with NL-SV1 become successively weaker as one moves to nuclei with higher isospin such as ${ }^{120} \mathrm{Sr}$ (Fig. 2(c)) and ${ }^{118} \mathrm{Kr}$ (Fig. 2(d)). It is worth making a special mention of the Kr nucleus. All the forces (except NL-SH) show a complete washing out of the shell effects. This stems from the fact that for ${ }^{118} \mathrm{Kr}$ the Fermi energy is very close to the continuum. The shell gap ceases to exist and consequently binding energy of an extra neutron is nearly zero. This implies that the shell effects for ${ }^{118} \mathrm{Kr}(N=82)$ are washed out. Such nuclei at the drip line $\left(\lambda_{n} \sim 0\right)$ are of little interest to the r-process as these nuclei can not bind an additional neutron. However, as the r-process path passes through $S_{n} \sim 2-4 \mathrm{MeV}$, it is seen (Figs. 2.a and 2.b) that nuclei which would contribute to the r-process show a persistence of stronger shell gaps in contrast to that seen for $\mathrm{Kr}$ and Sr. Figures 2(a) and 2(b) show that with NL-SV1 the shell effects for Mo $(\mathrm{Z}=42)$ and $\mathrm{Zr}(\mathrm{Z}=40)$ nuclei at $N=82$ are much stronger than with $\mathrm{SkP}$. For r-process nuclei with $Z>42$, the shell gaps are expected to be even larger. 


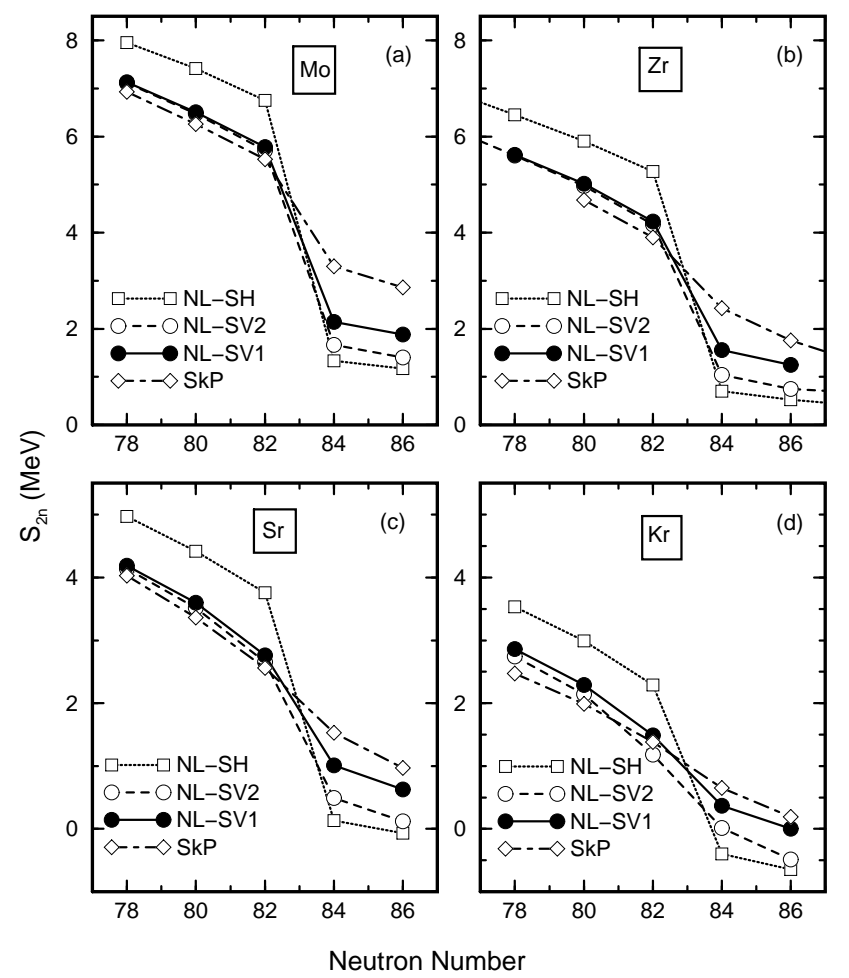

Figure 2. The RHB results for nuclei near the r-process path.

\section{CONCLUSION}

We have reproduced the shell effects about the waiting-point nucleus ${ }^{80} \mathrm{Zn}$ with the vector self-coupling of $\omega$-meson in the RHB theory. With this basis, it is shown that the shell effects near the r-process path about $N=82$ remain strong vis-a-vis a quenching exhibited by HFB+SkP. The quenching shown by SkP is not consistent with the available data. It is noteworthy that on the basis of the results with $\mathrm{SkP}$, a quenching has been requested for an improved fit to the global r-process abundances [1]. Since SkP is not able to reproduce the shell effects at the stability line and in the waiting-point region, an improved fit alone does not necessarily imply a shell quenching near the r-process path. Thus, alternative mechanisms for nucleosynthesis need to be looked into.

\section{REFERENCES}

1. K.-L. Kratz et al., Astrophys. J. 403, 216 (1993).

2. M. M. Sharma et al.,Phys. Rev. Lett.72, 1431 (1994).

3. J. Dobaczewski et al., Phys. Rev. Lett. 72, 981 (1994).

4. M. M. Sharma, A. R. Farhan and S. Mythili, Phys. Rev. C 61054306 (2000).

5. B. D. Serot and J. D. Walecka, Adv. Nucl. Phys. 16, 1 (1986).

6. A. R. Bodmer, Nucl. Phys. A 526, 703 (1991).

7. M. M. Sharma, M. A. Nagarajan and P. Ring, Phys. Lett. B 312, 377 (1993).

8. M. M. Sharma, in preparation (2000). 